139-03 - PROVA GRÁFICA - (15/06/05) Para: Volume 29, Número 3, 2005

\title{
MODIFICAÇÕES NAS FORMAS DE FÓSFORO DO SOLO APÓS EXTRAÇÕES SUCESSIVAS COM MEHLICH-1, MEHLICH-3 E RESINA TROCADORA DE ÂNIONS ${ }^{(1)}$
}

\author{
Luciano Colpo Gatiboni (2), J oão Kaminski(3) \& Danilo \\ Rheinheimer dos Santos ${ }^{(4)}$
}

\begin{abstract}
RESUMO
O fósforo encontra-se no solo em diversas formas, que variam de acordo com a natureza química dos compostos a que está ligado e à energia de ligação com estes. Assim, a labilidade das formas de P do solo é variável e os métodos de rotina utilizados para avaliação da disponibilidade para as plantas devem ser hábeis em dessorver as formas que têm capacidade de sustentar a absorção das plantas. $\mathbf{O}$ objetivo do presente trabal ho foi estudar o modo de ação de extratores por meio do acompanhamento das modificações ocorridas nas formas de $\mathbf{P}$ do solo após três e treze extrações sucessivas com os métodos Mehlich-1, Mehlich-3 e resina trocadora de ânions (RTA). Foram utilizadas amostras de um L atossolo Vermelho distroférrico típico cultivado sob sistema plantio direto e que recebeu, nos últi mos seis anos, doses anuais de $0,30,60,90$ e $120 \mathrm{~kg} \mathrm{ha}^{-1} \mathrm{P}_{2} \mathrm{O}_{5}$, totalizando $0,180,360,540$ e $720 \mathrm{~kg} \mathrm{ha}^{-1} \mathrm{P}_{2} \mathrm{O}_{5}$. Após as extrações sucessivas com os métodos, o solo remanescente foi seco em estufa e realizado o fracionamento químico do $P$, segundo o fracionamento de Hedley. Os resultados obtidos mostraram que os métodos Mehlich-1 e resina trocadora de ânions atuavam principalmente sobre as frações inorgânicas, sendo parte do $P$ dessorvido por esses extratores readsorvido aos colóides do solo, enquanto o método Mehlich-3 provocava a dessorção de $P$ tanto de formas inorgânicas como de orgânicas. Os métodos Mehlich-1, Mehlich-3 e RTA dessorveram o P de acordo com a labilidade no solo, extraindo, primeiramente, as formas mais lábeis e, posteriormente, as de menor labilidade.
\end{abstract}

Termos de indexação: fracionamento de fósforo, métodos de extração, disponibilidade de fósforo.

\footnotetext{
(1) Parte da Tese de Doutorado do primeiro autor. Trabalho financiado pelo CNPq/PRONEX e FAPERGS. Recebido para publicação em agosto de 2003 e aprovado em março de 2005.

(2) Professor da Universidade do Estado de Santa Catarina - UDESC. Rua Benjamin Constant 164-D, CEP 89806-070 Chapecó (SC). E-mail: gatiboni@udesc.br

(3) Professor do Departamento de Solos da Universidade Federal de Santa Maria - UFSM. CEP 97105-900 Santa Maria (RS). Email: kaminski@ccr.ufsm.br

(3) Professor do Departamento de Solos, UFSM. E-mail: danilor@smail.ufsm.br
} 


\title{
SUMMARY: ALTERATIONS IN SOIL PHOSPHORUS FORMS AFTER SUCCESSIVE EXTRACTIONS WITH MEHLICH-1, MEHLICH-3 AND ANION EXCHANGE RESIN METHODS
}

\begin{abstract}
Phosphorus is found in thesoil in several forms that vary according to the chemical nature of the compounds and the bound energy. Ther efore, methods for routine evaluation for the availability of $\mathrm{P}$ to plants should be able to extract the forms that sustain the plant uptake. This study evaluated the mode of action of Mehlich-1, Mehlich-3 and anion exchange resin (AER) methods by observing the modifications in soil $\mathrm{P}$ forms after three and thirteen successive extractions. Samples of a Rhodic Hapludox that had been cultivated under no-tillage and treated with annual doses of $0,30,60$, 90 , and $120 \mathrm{~kg} \mathrm{ha}^{-1} \mathrm{P}_{2} \mathrm{O}_{5}$, totalling $0,180,360,540$, and $720 \mathrm{~kg} \mathrm{ha}^{-1} \mathrm{P}_{2} \mathrm{O}_{5}$ throughout the previous six years were used. After the successive extractions, the remaining soil was oven-dried and submitted to soil $\mathrm{P}$ fractionati on according to Hedl ey's procedure. Results showed that M ehlich- 1 and AER methods extract mainly the inorganic forms, and a portion of desorbed $\mathrm{P}$ is readsorbed to the colloids. Mehlich-3 promotes the desorption of inorganic and organic P forms. Phosphorus desorption by Mehlich-1, Mehlich-3 and AER occurs according to its lability in the soil, extracting the most labile forms first, followed by those of lower lability.
\end{abstract}

Index terms: phosphorus fractionation, extraction methods, phosphorus availability.

\section{NTRODUÇÃO}

O P total do solo é particionado em diversas formas, variáveis com sua natureza química e com a energia da interação com os colóides. Quanto à natureza do composto, oP pode ser encontrado como $\mathrm{P}$ orgânico diéster, $\mathrm{P}$ orgâni co monoéster, $\mathrm{P}$ inorgânico em ligações com $\mathrm{Fe}, \mathrm{Al}, \mathrm{Ca}$, argilas silicatadas e óxidos, dentreoutras. J á, quantoà energia deligação, o $P$ pode estar associado a outros íons ou moléculas em ligações monodentadas, bidentadas e bionudeadas (Dalal, 1977; Parfitt, 1978).

Assim, as formas de $P$ do solo têm diferentes capacidades de dessorção e abastecimento da sol ução do solo, segundo sua natureza química e energia de ligação. $\mathrm{Na}$ avaliação da fertilidade do solo, independentemente da sua natureza química, o P é dividido de acordo com a facilidade de reposição do P da solução do solo, sendo dividido em formas lábeis, moderadamente lábeis e não-lábeis. Essa classificação é bastante acadêmica, mas ajuda no entendimento da dinâmica do P .

Para avaliar efetivamenteas formas de $\mathrm{P}$ do solo, pode-se utilizar a técnica de fracionamento proposta por Hedley et al. (1982), que usa, seqüencialmente, extratores de menor a maior poder de dessorção e permitem a separação das formas de $\mathrm{P}$ segundo sua natureza (orgânica ou inorgânica) e facilidade de dessorção (Cross \& Schlesinger, 1995).

Quando o objetivo da análise do $\mathrm{P}$ do solo é a estimativa da disponi bilidade para as plantas a curto prazo, têm-se utilizado extratores de menor poder de extração, que têm ação físico-química sobre as formas com menor energia que, em última análise, são as responsáveis pela capacidade de suprimento às plantas. No entanto, a eficiência dos extratores em predizer o estado da disponibilidade depende da sua seletividade sobre as formas inorgânicas ou orgânicas que predominam no grupo de solos avaliados (Fixen \& Grove, 1990; Silva \& Raij, 1999). E $m$ sol os carentes em $P$, formas de menor labilidade podem atuar no seu tamponamento, o que não se verifica em sol os com disponibilidade demédia a alta (Gatiboni, 2003). Por isso, há preocupação de entender o modo de ação dos extratores de rotina para avaliar sua capacidade de acesso a formas menos disponíveis quando do uso em sol os de menor disponibilidade.

Embora os procedimentos de rotina indicados para extratores de $\mathrm{P}$ estabel eçam uma só extração da amostra avaliada, al guns trabal hos mostram que a utilização de extrações sucessivas com esses mesmos métodos possibilita a ampliação do valor deP dessorvido do solo, ultrapassando os teores ditos lábeis, inferindo que os métodos de rotina, em extrações simples, acessam apenas parte do $\mathrm{P}$ potencialmente disponível às plantas (Campello et al., 1994; McKean \& Warren, 1996; Rheinheimer et al., 2000; Gatiboni et al ., 2002). Extrações sucessivas também permitem acompanhar, com maior segurança, as modificações provocadas pelos extratores nas formas de P do solo.

Rheinheimer et al. (2000), após realizarem 10 extrações sucessivas com resina trocadora deânions (RTA) em amostras de solos do Rio Grande do Sul e posterior fracionamento, observaram que, em média, $79 \%$ dofator quantidadefoi tamponado pelas frações inorgânicas extraídas com $\mathrm{NaOH} 0,1$ e 0,5 mol L-1 e $20 \%$ pela fração extraída com $\mathrm{NaHCO}_{3} 0,5 \mathrm{~mol} \mathrm{~L}^{-1}$, porém não avaliaram a dessorção das formas 
orgânicas. Com isso, os autores alertaram que o extrator $\mathrm{NaOH} 0,1 \mathrm{~mol} \mathrm{~L}^{-1}$, que estima formas de $\mathrm{P}$ de labilidade intermediária, poderia ser utilizado para avaliação do $\mathrm{P}$ potencial mente disponível.

Não há estudos sobre a ação dos extratores Mehlich-1 (Mehlich, 1953) e Mehlich-3 (Mehlich, 1984) sobre as formas orgânicas de $P$, embora se saiba que ajam principalmente sobre formas inorgânicas ligadas ao Ca e, em menor magnitude, àquelas ligadas ao Fe e Al (Fixen \& Grove, 1990). Assim, é necessário estudar a ação dos extratores sobre as formas orgânicas de $P$, podendo as de mai or labilidade atuar significativamentena reposição dos teores da solução do solo, quando da absorção pelas plantas (Adepetu \& Corey, 1976; Sharpley et al., 1987; Maroko et al., 1999).

O objetivo do presentetrabalhofoi estudar omodo de ação dos extratores por meio das modificações ocorridas nas formas de $P$ do solo após extrações sucessivas com os extratores Mehlich-1, Mehlich-3 e RTA.

\section{MATERIAL E MÉTODOS}

O experimento foi realizado no Laboratório de Química e Fertilidade do Solo da Universidade Federal deSanta Maria. Foram utilizadas amostras de um experimento do município de Santo Ângel o (RS), instalado em um solo classificado como L atossol o Vermel ho distroférrico típico (Streck et al., 2002), sob sistema plantio direto por seis anos. Foram col etadas amostras de solo da profundidade de $0-10 \mathrm{~cm}$ que receberam, anualmente, $0,30,60$, 90 e $120 \mathrm{~kg} \mathrm{ha}^{-1} \mathrm{P}_{2} \mathrm{O}_{5}$, total izando 0, 120, 360, 540 e $720 \mathrm{~kg} \mathrm{ha}^{-1} \mathrm{P}_{2} \mathrm{O}_{5}$ no momento da coleta das amostras. A pós a coleta, o sol o foi seco ao ar, moído, passado em peneira de mal ha $2 \mathrm{~mm}$ eacondicionado em sacos de polietileno. A análise do solo no momento da col eta seguiu os métodos descritos por Tedesco et al. (1995) e apresentou $640 \mathrm{~g} \mathrm{~kg}^{-1}$ de argila; $18 \mathrm{~g} \mathrm{~kg}^{-1}$ de matéria orgânica; $\mathrm{pH}-\mathrm{H}_{2} \mathrm{O}$ 4,8; índice SMP 5,7; 2,41 $\mathrm{cmol}_{\mathrm{C}} \mathrm{kg}^{-1}$ de $\mathrm{Ca}^{2+}$; $1,10 \mathrm{cmol}_{\mathrm{c}} \mathrm{kg}^{-1}$ de $\mathrm{Mg}^{2+} ; 0,85 \mathrm{cmol}_{\mathrm{c}} \mathrm{kg}^{-1}$ de $\mathrm{Al}^{3+} \mathrm{e}$ $310 \mathrm{mg} \mathrm{kg}^{-1}$ de $\mathrm{K}^{+}$.

As amostras desol o foram submetidas a extrações sucessivas com os métodos Mehlich-1 (Mehlich, 1953), Mehlich-3 (Mehlich, 1984) eRTA em lâminas descritas por Tedesco et al. (1995). As amostras foram arranjadas em duplicata, tendo sido submetidas a três ou a treze extrações sucessivas, quando os teores extraídos em cada extração tornaram-se baixos e constantes. Para as extrações, $1,000 \mathrm{~g}$ de solo foi acondicionado em tubos de centrífuga com tampa rosca e procedido o protocolo específico a cada método. Após o tempo de extração, as amostras foram centrifugadas a $6.000 \mathrm{rpm}$, analisando-se o teor de $\mathrm{P}$ no extrato e submetendo- se o sol o a uma nova extração. Não houve intervalo de descanso da amostra de sol o entre uma extração sucessiva e outra, uma vez que, não se observaram diferenças na dessorção deP entre diferentes tempos de repouso das amostras (Campello et al., 1994; Rheinheimer et al., 2000). Informações adicionais sobre o procedimento de extrações sucessivas estão descritas em maiores detalhes em Gatiboni et al. (2002).

A pós a última extração sucessiva prevista, o solo remanescente no tubo foi seco em estufa a $55^{\circ} \mathrm{C}$ e o solo retirado do tubo e moído em gral de porcelana. O solo recuperado foi submetido ao fracionamento químico do $\mathrm{P}$, segundo método desenvolvido por Hedley et al. (1982), com as modificações propostas por Condron et al. (1985), descritas a seguir. Amostras de $0,500 \mathrm{~g}$ foram extraídas, seqüencialmente, com resina trocadora deânions (placas AR 103 QDP 434, Ionics Inc.), $\mathrm{NaHCO}_{3} 0,5 \mathrm{~mol} \mathrm{~L}^{-1}, \mathrm{NaOH} \mathrm{0,1} \mathrm{mol} \mathrm{L-1,}$ $\mathrm{HCl} 1,0 \mathrm{~mol} \mathrm{~L}^{-1}$ e NaOH $0,5 \mathrm{~mol} \mathrm{~L}^{-1}$. Após a extração com $\mathrm{NaOH} 0,5 \mathrm{~mol} \mathrm{~L}^{-1}$, o sol o remanescente foi seco em estufa esubmeti do à digestão com $\mathrm{H}_{2} \mathrm{SO}_{4}+\mathrm{H}_{2} \mathrm{O}_{2}$ $+\mathrm{MgCl}_{2}$ saturado (Brookes \& Powlson, 1981). O P inorgânico dos extratos alcalinos de $\mathrm{NaHCO}_{3} \mathrm{e}$ $\mathrm{NaOH}$ foi analisado pelo método de Dick \& Tabatabai (1977). Nos extratos alcalinos, foi determinado o $\mathrm{P}$ total por digestão com persulfato de amônio + ácido sulfúrico em autoclave (USEPA, 1971), enquanto oP orgâni co foi obtido pela diferença entre $\mathrm{P}$ total e o inorgânico. $\mathrm{O} P$ dos extratos ácidos foi determinado segundo o método de Murphy \& Riley (1962).

O delineamento experimental utilizado foi um fatorial $5 \times 3 \times 3$ inteiramente casualizado e com quatro repetições, sendo cinco doses de $P(0,120$, 360,540 e $720 \mathrm{~kg} \mathrm{ha}^{-1} \mathrm{P}_{2} \mathrm{O}_{5}$ ): os métodos de extrações sucessivas (Mehlich-1, Mehlich-3 eRTA), eo número de extrações sucessivas (zero, três ou treze). Os dados foram submetidos à análise da variância e, quando dos efeitos e interações significativos, as médias foram comparadas pelo teste da diferença mínima significativa (DMS) com $5 \%$.

\section{RESULTADOS E DISCUSSÃO}

Os teores de $\mathrm{P}$ nas diferentes formas ou frações após as extrações sucessivas com Mehlich-1 são mostrados no quadro 1. Pode-se observar quea forma mais lábil de $\mathrm{P}$ inorgânico $\left(\mathrm{Pi}_{\mathrm{RTA}}\right)$, que é extraída por resina de troca aniônica no fracionamento, foi praticamente toda dessorvida já com três extrações, não sendo modificada atéa décima terceira extração. I sso indica que, após a terceira, as extrações subseqüentes dessorveram $P$ de frações de menor labilidade. As extrações sucessivas com Mehlich-1 não provocaram modificações na fração $\mathrm{Pi}_{\text {bic }}$ dos tratamentos com menor adição de $\mathrm{P}(0,180$ e $360 \mathrm{~kg} \mathrm{ha}^{-1} \mathrm{P}_{2} \mathrm{O}_{5}$ ), mas provocaram diminuição dos 
teores nos tratamentos com maiores doses de $\mathrm{P}$ adicionadas (540 e $720 \mathrm{~kg} \mathrm{ha}^{-1} \mathrm{P}_{2} \mathrm{O}_{5}$ ). Como o P extraído por bicarbonato também éreconhedido como uma forma lábil (Cross \& Schlesinger, 1995), o comportamento observado mostra que a dessorção é proporcional à quantidade de $\mathrm{P}$ acumulada pela adubação. Esse comportamento também foi observado na fração inorgânica extraída com $\mathrm{NaOH}$ $0,1 \mathrm{~mol} \mathrm{~L}^{-1}\left(\mathrm{Pi}_{\text {hid }}\right)$, para a qual nas menores doses não foi observada diminuição dos teores após as extrações sucessivas, ocorrendo apenas nos tratamentos com 540 e $720 \mathrm{~kg} \mathrm{ha}^{-1} \mathrm{P}_{2} \mathrm{O}_{5}$ (Quadro 1).

A fração extraída com HCl 1 mol L-1 $\left(\mathrm{Pi}_{\mathrm{HCl}}\right)$, tida como proveniente de compostos ligados ao Ca (Cross \& Schlesinger, 1995), não foi atingi da pelas extrações sucessivas com Mehlich-1 (Quadro 1 ), o que aparentemente contradiz as afirmações de Fixen \& Grove (1990), que dizem que este extrator extrai preferencialmente compostos de P-Ca. Todavia, como o solo utilizado apresenta avançado grau de intemperismo, é provável que a presença deste tipo de composto já tenha sido muito reduzida.

A forma inorgânica de $\mathrm{P}$ extraída com $\mathrm{NaOH}$ $0,5 \mathrm{~mol} \mathrm{~L}^{-1}\left(\mathrm{Pi}_{\text {hid05 }}\right)$ foi pouco modificada pelas extrações sucessivas com Mehlich-1 (Quadro 1), sendo observado apenas um pequeno aumento de $\mathrm{P}$, principal menteapós três extrações sucessivas, no tratamento testemunha $\left(0 \mathrm{~kg} \mathrm{ha}-1 \mathrm{P}_{2} \mathrm{O}_{5}\right)$, o que pode ser devido à readsorção de $\mathrm{P}$ proveniente de outras frações por causa da alta avidez por P dos sítios de adsorção neste tratamento. A readsorção de $\mathrm{P}$ durante o processo de extração do Mehlich-1 é um fenômeno conhecido, no qual, após a dessorção, parte do extraído retorna aos sítios de adsorção pelo aumento da avidez destes (Cajuste \& Kussow, 1974; Novais \& Smyth, 1999).

\section{Quadro 1. Teores de diferentes formas de fósforo no solo antes e depois de três e treze extrações sucessivas com Mehlich-1}

\begin{tabular}{|c|c|c|c|c|c|c|}
\hline \multirow{2}{*}{ Forma de fósforo } & \multirow{2}{*}{$\begin{array}{c}\text { Número de } \\
\text { extração } \\
\text { sucessiva }\end{array}$} & \multicolumn{5}{|c|}{ Dose de $\mathrm{P}_{2} \mathrm{O}_{5}\left(\mathrm{~kg} \mathrm{ha}^{-1}\right)$} \\
\hline & & $\mathbf{0}$ & 180 & 360 & 540 & 720 \\
\hline & & \multicolumn{5}{|c|}{$-\mathrm{mg} \mathrm{kg}^{-1}$} \\
\hline & & \multicolumn{5}{|c|}{ Formas inorgânicas } \\
\hline $\operatorname{Pi}_{\text {RTA }}(\text { RTA })^{(1)}$ & $\begin{array}{l}0^{(3)} \\
3 \\
13\end{array}$ & $\begin{array}{l}7 a^{(2)} \\
1 \mathrm{~b} \\
1 \mathrm{~b}\end{array}$ & $\begin{array}{r}11 \mathrm{a} \\
1 \mathrm{~b} \\
1 \mathrm{~b}\end{array}$ & $\begin{array}{r}13 \mathrm{a} \\
1 \mathrm{~b} \\
1 \mathrm{~b}\end{array}$ & $\begin{array}{r}23 a \\
3 b \\
2 b\end{array}$ & $\begin{array}{r}29 a \\
3 b \\
2 b\end{array}$ \\
\hline $\mathrm{Pi}_{\text {bic }}\left(\mathrm{NaHCO}_{3} 0,5 \mathrm{~mol} \mathrm{~L}^{-1}\right)$ & $\begin{array}{r}0 \\
3 \\
13\end{array}$ & $\begin{array}{l}5 a \\
7 a \\
8 a\end{array}$ & $\begin{array}{c}7 \mathrm{~b} \\
10 \mathrm{a} \\
9 \mathrm{ab}\end{array}$ & $\begin{array}{r}8 \mathrm{c} \\
11 \mathrm{a} \\
10 \mathrm{~b}\end{array}$ & $\begin{array}{l}16 \mathrm{~b} \\
18 \mathrm{a} \\
14 \mathrm{c}\end{array}$ & $\begin{array}{l}18 \mathrm{a} \\
19 \mathrm{a} \\
14 \mathrm{~b}\end{array}$ \\
\hline $\mathrm{Pi}_{\text {hid }}\left(\mathrm{NaOH} 0,1 \mathrm{~mol} \mathrm{~L}^{-1}\right)$ & $\begin{array}{r}0 \\
3 \\
13\end{array}$ & $\begin{array}{l}53 a \\
75 a \\
66 a\end{array}$ & $\begin{array}{l}58 c \\
82 \mathrm{a} \\
70 \mathrm{~b}\end{array}$ & $\begin{array}{l}65 a \\
83 a \\
79 a\end{array}$ & $\begin{array}{r}97 \mathrm{~b} \\
112 \mathrm{a} \\
81 \mathrm{~b}\end{array}$ & $\begin{array}{r}110 a \\
116 a \\
92 b\end{array}$ \\
\hline $\mathrm{Pi}_{\mathrm{HCl}}\left(\mathrm{HCl} 1,0 \mathrm{~mol} \mathrm{~L}^{-1}\right)$ & $\begin{array}{r}0 \\
3 \\
13\end{array}$ & $\begin{array}{r}8 a \\
10 a \\
10 a\end{array}$ & $\begin{array}{l}11 \mathrm{a} \\
12 \mathrm{a} \\
11 \mathrm{a}\end{array}$ & $\begin{array}{l}11 \mathrm{a} \\
12 \mathrm{a} \\
11 \mathrm{a}\end{array}$ & $\begin{array}{l}13 \mathrm{~b} \\
15 \mathrm{a} \\
13 \mathrm{~b}\end{array}$ & $\begin{array}{l}13 b \\
16 a \\
13 b\end{array}$ \\
\hline $\mathrm{Pi}_{\text {hid05 }}\left(\mathrm{NaOH} 0,5 \mathrm{~mol} \mathrm{~L}^{-1}\right)$ & $\begin{array}{r}0 \\
3 \\
13\end{array}$ & $\begin{array}{r}79 \mathrm{~b} \\
118 \mathrm{a} \\
93 \mathrm{~b}\end{array}$ & $\begin{array}{r}83 a \\
107 a \\
94 a\end{array}$ & $\begin{array}{r}92 a \\
102 a \\
107 a\end{array}$ & $\begin{array}{r}101 a \\
99 a \\
103 a\end{array}$ & $\begin{array}{r}111 \mathrm{a} \\
94 \mathrm{a} \\
103 \mathrm{a}\end{array}$ \\
\hline \multirow[t]{2}{*}{$\mathrm{P}_{\text {res }}\left(\mathrm{H}_{2} \mathrm{SO}_{4}+\mathrm{H}_{2} \mathrm{O}_{2}+\mathrm{MgCl}_{2}\right)$} & $\begin{array}{r}0 \\
3 \\
13\end{array}$ & $\begin{array}{l}433 a \\
361 b \\
334 b\end{array}$ & $\begin{array}{l}451 \mathrm{a} \\
385 \mathrm{~b} \\
389 \mathrm{~b}\end{array}$ & $\begin{array}{l}443 a \\
421 a \\
395 a\end{array}$ & $\begin{array}{l}451 a \\
428 a \\
424 a\end{array}$ & $\begin{array}{l}456 a \\
414 a \\
421 a\end{array}$ \\
\hline & & \multicolumn{5}{|c|}{ Formas orgânicas } \\
\hline $\left.\mathrm{PObic}_{\left(\mathrm{NaHCO}_{3}\right.} 0,5 \mathrm{~mol} \mathrm{~L}^{-1}\right)$ & $\begin{array}{r}0 \\
3 \\
13\end{array}$ & $\begin{array}{l}14 b \\
31 a \\
30 a\end{array}$ & $\begin{array}{l}14 b \\
35 a \\
33 a\end{array}$ & $\begin{array}{l}15 \mathrm{~b} \\
38 \mathrm{a} \\
35 \mathrm{a}\end{array}$ & $\begin{array}{l}14 \mathrm{~b} \\
35 \mathrm{a} \\
36 \mathrm{a}\end{array}$ & $\begin{array}{l}14 b \\
34 a \\
37 a\end{array}$ \\
\hline POhid $\left(\mathrm{NaOH} 0,1 \mathrm{~mol} \mathrm{~L}^{-1}\right)$ & $\begin{array}{r}0 \\
3 \\
13\end{array}$ & $\begin{array}{l}89 a \\
67 c \\
77 b\end{array}$ & $\begin{array}{r}93 \mathrm{a} \\
94 \mathrm{a} \\
102 \mathrm{a}\end{array}$ & $\begin{array}{l}87 a \\
97 a \\
87 a\end{array}$ & $\begin{array}{l}100 a \\
109 a \\
104 a\end{array}$ & $\begin{array}{l}104 a \\
110 a \\
114 a\end{array}$ \\
\hline POhid05 (NaOH 0,5 mol L-1) & $\begin{array}{r}0 \\
3 \\
13\end{array}$ & $\begin{array}{l}36 a \\
17 a \\
28 a\end{array}$ & $\begin{array}{l}49 a \\
44 a b \\
37 b\end{array}$ & $\begin{array}{l}42 \mathrm{a} \\
34 \mathrm{~b} \\
25 \mathrm{c}\end{array}$ & $\begin{array}{l}35 \mathrm{~b} \\
57 \mathrm{a} \\
31 \mathrm{~b}\end{array}$ & $\begin{array}{l}25 a \\
57 a \\
31 a\end{array}$ \\
\hline
\end{tabular}

(1) Entre parênteses é mostrado o extrator utilizado para cada forma de fósforo. ${ }^{(2)}$ Médias seguidas da mesma letra entre as extrações e dentro da mesma forma de fósforo não diferem entre si pelo teste DMS $(P<0,05)$. ${ }^{(3)}$ Solo original sem extrações sucessivas previamente ao fracionamento. 
Nos tratamentos 0 e $180 \mathrm{~kg} \mathrm{ha}-1 \mathrm{P}_{2} \mathrm{O}_{5}$, as extrações sucessivas com Mehlich-1 provocaram dessorção de $P$ da fração residual $\left(P_{\text {res }}\right)$, indicando que, nestes tratamentos, pela baixa quantidade de P acumulada nas formas delabilidadeintermediária, a fração de maior recalcitrância foi atingida pelo extrator (Quadro 1). J á, nos tratamentos com maior adição de $P$, o $P_{\text {res }}$ não foi modificado, indicando que o $\mathrm{P}$ dessorvido pelas extrações sucessivas foi proveniente de frações mais lábeis que o $\mathrm{P}_{\text {res }}\left(\mathrm{Pi}_{\mathrm{RTA}}\right.$, $\mathrm{Pi}_{\text {bic }}$ e $\mathrm{Pi}_{\text {hid }}$ ). Com isso, parece que o Mehlich-1 dessorve $\mathrm{P}$ das diferentes formas obedecendo sua labilidade ou capacidade de dessorção, mas, quando a quantidade de $P$ nas formas mais lábeis é baixa, a fração residual é afetada, como observado por Gatiboni et al. (2002).

J á o teor de $\mathrm{P}$ orgânico extraído com $\mathrm{NaHCO}_{3}$ 0,5 mol L-1 $\left(\mathrm{Po}_{\text {bic }}\right)$ aumentou em relação ao teor original (Quadro 1), enquanto o P orgânico extraído com NaOH 0,1 mol L-1 (Po hid $\left._{1}\right)$ e $\mathrm{NaOH} 0,5 \mathrm{~mol} \mathrm{~L}^{-1}$ $\left(\mathrm{PO}_{\text {hid05 }}\right)$, principalmente o segundo, apresentou diminuição após as extrações sucessivas. I sso pode ter ocorrido porque as extrações sucessivas causaram a destruição dos mi croagregados do solo, retirando a proteção física da matéria orgânica e expondo-a para o ataque do bicarbonato, visto que setrata de um sol o muito argiloso, rico em óxidos e, portanto, com alta capacidade de proteção dos compostos orgânicos (Bayer, 1996).

Com relação ao extrator Mehlich-3 (Quadro 2), pode ser observado que a depleção do $\mathrm{Pi}_{\mathrm{RTA}}$ após as três primeiras extrações não foi tão drástica como a ocorrida no Mehlich-1, sendo progressiva com o aumento das extrações. O teor na forma $\mathrm{Pi}_{\text {bic }}$ comportou-se de modo similar ao observado para o Mehlich-1, havendo pequeno aumento nos tratamentos com menores doses de $\mathrm{P}(0,180 \mathrm{e}$ $360 \mathrm{~kg} \mathrm{ha}^{-1} \mathrm{P}_{2} \mathrm{O}_{5}$ ) e depleção de $\mathrm{P}$ nos tratamentos 540 e $720 \mathrm{~kg} \mathrm{ha}^{-1} \mathrm{P}_{2} \mathrm{O}_{5}$ (Quadro 2).

Foi observado aumento do teor de $\mathrm{Pi}_{\text {hid }}$ após três extrações com Mehlich-3 e diminuição após treze extrações nos tratamentos 0,180 e $360 \mathrm{~kg} \mathrm{ha}^{-1} \mathrm{P}_{2} \mathrm{O}_{5}$,

\section{Quadro 2. Teores de diferentes formas de fósforo no solo antes e depois de três e treze extrações sucessivas} com Mehlich-3

\begin{tabular}{|c|c|c|c|c|c|c|}
\hline \multirow{2}{*}{ F orma de fósforo } & \multirow{2}{*}{$\begin{array}{l}\text { Número de } \\
\text { extração } \\
\text { sucessiva }\end{array}$} & \multicolumn{5}{|c|}{ Dose de $\mathrm{P}_{2} \mathrm{O}_{5}\left(\mathrm{~kg} \mathrm{ha}^{-1}\right)$} \\
\hline & & $\mathbf{0}$ & 180 & 360 & 540 & 720 \\
\hline & & \multicolumn{5}{|c|}{$-\mathrm{mg} \mathrm{kg}^{-1}$} \\
\hline & & \multicolumn{5}{|c|}{ Formas inorgânicas } \\
\hline $\mathrm{Pi}_{\mathrm{RTA}}(\mathrm{RTA})^{(1)}$ & $\begin{array}{l}0^{(3)} \\
3 \\
13\end{array}$ & $\begin{array}{l}7 \mathrm{a}^{(2)} \\
4 \mathrm{~b} \\
3 \mathrm{c}\end{array}$ & $\begin{array}{r}11 \mathrm{a} \\
5 \mathrm{~b} \\
4 \mathrm{~b}\end{array}$ & $\begin{array}{r}13 a \\
6 b \\
5 b\end{array}$ & $\begin{array}{r}23 a \\
10 b \\
5 c\end{array}$ & $\begin{array}{r}29 a \\
12 b \\
4 c\end{array}$ \\
\hline $\mathrm{Pi}_{\text {bic }}\left(\mathrm{NaHCO}_{3} 0,5 \mathrm{~mol} \mathrm{~L}^{-1}\right)$ & $\begin{array}{r}0 \\
3 \\
13\end{array}$ & $\begin{array}{l}5 \mathrm{~b} \\
8 \mathrm{a} \\
9 \mathrm{a}\end{array}$ & $\begin{array}{r}7 \mathrm{~b} \\
11 \mathrm{a} \\
11 \mathrm{a}\end{array}$ & $\begin{array}{r}8 \mathrm{~b} \\
11 \mathrm{a} \\
10 \mathrm{a}\end{array}$ & $\begin{array}{l}16 \mathrm{~b} \\
19 \mathrm{a} \\
14 \mathrm{c}\end{array}$ & $\begin{array}{l}18 \mathrm{a} \\
20 \mathrm{a} \\
14 \mathrm{~b}\end{array}$ \\
\hline $\mathrm{Pi}_{\text {hid }}\left(\mathrm{NaOH} 0,1 \mathrm{~mol} \mathrm{~L}^{-1}\right)$ & $\begin{array}{r}0 \\
3 \\
13\end{array}$ & $\begin{array}{l}53 \mathrm{~b} \\
80 \mathrm{a} \\
54 \mathrm{~b}\end{array}$ & $\begin{array}{l}58 c \\
90 a \\
70 b\end{array}$ & $\begin{array}{l}65 \mathrm{~b} \\
93 \mathrm{a} \\
74 \mathrm{~b}\end{array}$ & $\begin{array}{c}97 a b \\
113 a \\
76 b\end{array}$ & $\begin{array}{r}110 a \\
117 a \\
73 b\end{array}$ \\
\hline $\mathrm{Pi}_{\mathrm{HCl}}\left(\mathrm{HCl} 1,0 \mathrm{~mol} \mathrm{~L}^{-1}\right)$ & $\begin{array}{r}0 \\
3 \\
13\end{array}$ & $\begin{array}{r}8 a \\
12 a \\
10 a\end{array}$ & $\begin{array}{l}11 \mathrm{~b} \\
14 \mathrm{a} \\
11 \mathrm{~b}\end{array}$ & $\begin{array}{l}11 a \\
14 a \\
12 a\end{array}$ & $\begin{array}{l}13 \mathrm{~b} \\
16 \mathrm{a} \\
12 \mathrm{~b}\end{array}$ & $\begin{array}{l}13 a \\
16 a \\
12 a\end{array}$ \\
\hline $\mathrm{Pi}_{\text {hid05 }}\left(\mathrm{NaOH} 0,5 \mathrm{~mol} \mathrm{~L}^{-1}\right)$ & $\begin{array}{r}0 \\
3 \\
13\end{array}$ & $\begin{array}{r}79 a \\
93 a \\
110 a\end{array}$ & $\begin{array}{r}83 a \\
95 a \\
114 a\end{array}$ & $\begin{array}{r}92 a \\
111 a \\
125 a\end{array}$ & $\begin{array}{l}101 c \\
116 b \\
124 a\end{array}$ & $\begin{array}{l}111 b \\
117 a \\
126 a\end{array}$ \\
\hline \multirow[t]{2}{*}{$\mathrm{P}_{\mathrm{res}}\left(\mathrm{H}_{2} \mathrm{SO}_{4}+\mathrm{H}_{2} \mathrm{O}_{2}+\mathrm{MgCl}_{2}\right)$} & $\begin{array}{r}0 \\
3 \\
13\end{array}$ & $\begin{array}{l}433 a \\
447 a \\
412 a\end{array}$ & $\begin{array}{l}451 a \\
451 a \\
406 a\end{array}$ & $\begin{array}{l}443 a \\
441 a \\
434 a\end{array}$ & $\begin{array}{l}451 a \\
440 a \\
442 a\end{array}$ & $\begin{array}{l}456 a \\
438 a \\
436 a\end{array}$ \\
\hline & & \multicolumn{5}{|c|}{ Formas orgânicas } \\
\hline $\mathrm{PO}_{\text {bic }}\left(\mathrm{NaHCO}_{3} 0,5 \mathrm{~mol} \mathrm{~L}^{-1}\right)$ & $\begin{array}{r}0 \\
3 \\
13\end{array}$ & $\begin{array}{l}14 b \\
25 a \\
24 a\end{array}$ & $\begin{array}{l}14 a \\
30 a \\
25 a\end{array}$ & $\begin{array}{l}15 a \\
26 a \\
26 a\end{array}$ & $\begin{array}{l}14 b \\
29 a \\
27 a\end{array}$ & $\begin{array}{l}14 b \\
28 a \\
28 a\end{array}$ \\
\hline POhid (NaOH 0,1 mol L-1) & $\begin{array}{r}0 \\
3 \\
13\end{array}$ & $\begin{array}{l}89 a \\
61 \mathrm{~b} \\
79 \mathrm{a}\end{array}$ & $\begin{array}{l}93 a \\
73 a \\
84 a\end{array}$ & $\begin{array}{l}87 a \\
81 a \\
79 a\end{array}$ & $\begin{array}{r}100 \mathrm{a} \\
74 \mathrm{~b} \\
96 \mathrm{a}\end{array}$ & $\begin{array}{r}104 a \\
73 a \\
97 a\end{array}$ \\
\hline Ponid05 (NaOH 0,5 mol L-1) & $\begin{array}{r}0 \\
3 \\
13\end{array}$ & $\begin{array}{l}36 a \\
40 a \\
11 a\end{array}$ & $\begin{array}{l}49 \mathrm{a} \\
45 \mathrm{a} \\
17 \mathrm{~b}\end{array}$ & $\begin{array}{l}42 \mathrm{a} \\
20 \mathrm{~b} \\
13 \mathrm{~b}\end{array}$ & $\begin{array}{r}35 a \\
27 a \\
9 b\end{array}$ & $\begin{array}{r}25 a \\
18 a \\
6 b\end{array}$ \\
\hline
\end{tabular}

(1) Entre parênteses é mostrado o extrator utilizado para cada forma de fósforo. ${ }^{(2)}$ Médias seguidas da mesma letra entre as extrações e dentro da mesma forma de fósforo não diferem entre si pelo teste DMS $(P<0,05)$. ${ }^{(3)}$ Solo original sem extrações sucessivas previamente ao fracionamento. 
podendo inferir que, inicial mente, houve readsor ção de $P$ nessa forma e, posteriormente, depleção (Quadro 2). J á nos tratamentos 540 e $720 \mathrm{~kg} \mathrm{ha}^{-1}$ $\mathrm{P}_{2} \mathrm{O}_{5}$, pelos maiores teores disponíveis, sempre houve depleção de $\mathrm{P}$, similarmente ao observado no extrator Mehlich-1.

I gualmente ao observado com Mehlich-1, o uso do Mehlich-3 não afetou as formas $\mathrm{Pi}_{\mathrm{HCl}} \mathrm{ePi}_{\text {hid05. }} \mathrm{O}$ $P_{\text {res }}$ também não foi modificado pelo Mehlich-3, mesmonos tratamentos 0 e $180 \mathrm{~kg} \mathrm{ha}{ }^{-1} \mathrm{P}_{2} \mathrm{O}_{5}$, diferindo do observado com o uso do Mehlich-1, o que indica que este último é mais agressivo que o Mehlich-3.

Com o uso do Mehlich-3, as formas orgânicas de $\mathrm{P}\left(\mathrm{Po}_{\mathrm{bic}}, \mathrm{Po}_{\text {hid }}\right.$ e $\left.\mathrm{PO}_{\text {hid05 }}\right)$ foram modificadas de maneira diferente do ocorrido para o Mehlich-1. Embora também tenha sido observado aumento dos teores de $\mathrm{PO}_{\mathrm{bic}}$ após as extrações sucessivas de acordo com a desagregação do solo, foi observada grande depleção do $\mathrm{PO}_{\text {hid05, }}$ cuja diferença de teores foi maior que o aumento observado no $\mathrm{PO}_{\mathrm{bic}}$. I sso indica que parte do Po total foi mineralizado durante as extrações sucessivas, o que pode ser devido à presença deEDTA na solução extratora do Mehlich-3, que aumenta a capacidade de dessorção de compostos orgânicos (Tate \& N ewman, 1982).

O fracionamento não detectou depleção na forma $\mathrm{Pi}_{\mathrm{RTA}}$ após as extrações sucessivas nos tratamentos 0,180 e $360 \mathrm{~kg} \mathrm{ha}^{-1} \mathrm{P}_{2} \mathrm{O}_{5}$ e detectou apenas uma pequena depleção nos tratamentos 540 e $720 \mathrm{~kg} \mathrm{ha}^{-1}$ $\mathrm{P}_{2} \mathrm{O}_{5}$ (Quadro 3). No entanto, Gatiboni et al. (2002) verificaram que, durante as extrações sucessivas com RTA, omontante deP extraído em cada extração baixou drasticamente a partir da quarta extração, chegando a quase zero na décima terceira extração. Assim, o restabelecimento dos altos teores observados na fração $\mathrm{Pi}_{\text {RTA }}$ do fracionamento pode ter ocorrido por causa da secagem da amostra, realizada entre a última extração sucessiva e o fracionamento, que, segundo Barrow (1987), pode desestabilizar as ligações químicas, facilitando a dessor ção, embora isso não tenha sido observado por Campello et al. (1994) e Rheinheimer et al. (2000).

A reposição do teor na fração $\mathrm{Pi}_{\mathrm{RTA}}$ foi feita pela fração $\mathrm{Pi}_{\text {bic }}$ pois esta foi completamente dessorvida já na terceira extração sucessiva. A completa depleção do $\mathrm{Pi}_{\text {bic }}$ e aumento do $\mathrm{Pi}_{\mathrm{RTA}}$ indica que os

Quadro 3. Teores de diferentes formas de fósforo no solo antes e depois de três e treze extrações sucessivas com resina trocadora de ânions

\begin{tabular}{|c|c|c|c|c|c|c|}
\hline \multirow{2}{*}{ Forma de fósforo } & \multirow{2}{*}{$\begin{array}{l}\text { Número de } \\
\text { extração } \\
\text { sucessiva }\end{array}$} & \multicolumn{5}{|c|}{ Dose de $\mathrm{P}_{2} \mathrm{O}_{5}(\mathrm{~kg} \mathrm{ha-1)}$} \\
\hline & & $\mathbf{0}$ & 180 & 360 & 540 & 720 \\
\hline & & 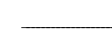 & 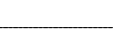 & $\mathrm{mg} \mathrm{kg}^{-1}$ & 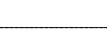 & 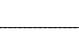 \\
\hline & & \multicolumn{5}{|c|}{ Formas inorgânicas } \\
\hline $\mathrm{Pi}_{\mathrm{RTA}}(\mathrm{RTA})^{(1)}$ & $\begin{array}{l}0(3) \\
3 \\
13\end{array}$ & $\begin{array}{l}7 b^{(2)} \\
7 b \\
9 a\end{array}$ & $\begin{array}{l}11 a \\
12 a \\
11 a\end{array}$ & $\begin{array}{l}13 a \\
13 a \\
12 a\end{array}$ & $\begin{array}{l}23 \mathrm{a} \\
18 \mathrm{~b} \\
16 \mathrm{~b}\end{array}$ & $\begin{array}{l}29 a \\
19 b \\
15 b\end{array}$ \\
\hline $\mathrm{Pi}_{\text {bic }}\left(\mathrm{NaHCO}_{3} 0,5 \mathrm{~mol} \mathrm{~L}^{-1}\right)$ & $\begin{array}{r}0 \\
3 \\
13\end{array}$ & $\begin{array}{l}5 \mathrm{a} \\
0 \mathrm{~b} \\
0 \mathrm{~b}\end{array}$ & $\begin{array}{l}7 \mathrm{a} \\
0 \mathrm{~b} \\
0 \mathrm{~b}\end{array}$ & $\begin{array}{l}8 \mathrm{a} \\
0 \mathrm{~b} \\
0 \mathrm{~b}\end{array}$ & $\begin{array}{r}16 \mathrm{a} \\
0 \mathrm{~b} \\
0 \mathrm{~b}\end{array}$ & $\begin{array}{r}18 \mathrm{a} \\
1 \mathrm{~b} \\
0 \mathrm{~b}\end{array}$ \\
\hline $\mathrm{Pi}_{\text {hid }}\left(\mathrm{NaOH} 0,1 \mathrm{~mol} \mathrm{~L}^{-1}\right)$ & $\begin{array}{r}0 \\
3 \\
13\end{array}$ & $\begin{array}{l}53 \mathrm{a} \\
45 \mathrm{~b} \\
39 \mathrm{c}\end{array}$ & $\begin{array}{l}58 \mathrm{a} \\
51 \mathrm{~b} \\
45 \mathrm{c}\end{array}$ & $\begin{array}{l}65 \mathrm{a} \\
52 \mathrm{~b} \\
45 \mathrm{c}\end{array}$ & $\begin{array}{l}97 \mathrm{a} \\
68 \mathrm{~b} \\
48 \mathrm{c}\end{array}$ & $\begin{array}{r}110 \mathrm{a} \\
71 \mathrm{~b} \\
50 \mathrm{c}\end{array}$ \\
\hline $\mathrm{Pi}_{\mathrm{HCl}}\left(\mathrm{HCl} 1,0 \mathrm{~mol} \mathrm{~L}^{-1}\right)$ & $\begin{array}{r}0 \\
3 \\
13\end{array}$ & $\begin{array}{r}8 a \\
10 a \\
10 a\end{array}$ & $\begin{array}{l}11 \mathrm{c} \\
13 \mathrm{a} \\
12 \mathrm{~b}\end{array}$ & $\begin{array}{l}11 \mathrm{c} \\
15 \mathrm{a} \\
14 \mathrm{~b}\end{array}$ & $\begin{array}{l}13 c \\
17 a \\
15 b\end{array}$ & $\begin{array}{l}13 \mathrm{~b} \\
17 \mathrm{a} \\
15 \mathrm{a}\end{array}$ \\
\hline $\mathrm{Pi}_{\text {hid05 }}\left(\mathrm{NaOH} 0,5 \mathrm{~mol} \mathrm{~L}^{-1}\right)$ & $\begin{array}{r}0 \\
3 \\
13\end{array}$ & $\begin{array}{r}79 a \\
107 a \\
107 a\end{array}$ & $\begin{array}{r}83 a \\
116 a \\
107 a\end{array}$ & $\begin{array}{l}92 \mathrm{~b} \\
124 \mathrm{a} \\
115 \mathrm{ab}\end{array}$ & $\begin{array}{l}101 \mathrm{~b} \\
128 \mathrm{a} \\
122 \mathrm{a}\end{array}$ & $\begin{array}{l}111 a \\
126 a \\
129 a\end{array}$ \\
\hline \multirow[t]{2}{*}{$\mathrm{P}_{\text {res }}\left(\mathrm{H}_{2} \mathrm{SO}_{4}+\mathrm{H}_{2} \mathrm{O}_{2}+\mathrm{MgCl}_{2}\right)$} & $\begin{array}{r}0 \\
3 \\
13\end{array}$ & $\begin{array}{l}433 a \\
408 a \\
405 a\end{array}$ & $\begin{array}{l}451 a \\
443 a \\
393 a\end{array}$ & $\begin{array}{l}443 a \\
413 a \\
399 a\end{array}$ & $\begin{array}{l}451 a \\
423 b \\
401 c\end{array}$ & $\begin{array}{l}456 a \\
419 b \\
388 c\end{array}$ \\
\hline & & \multicolumn{5}{|c|}{ Formas orgânicas } \\
\hline $\mathrm{Pobic}_{\text {bic }}\left(\mathrm{NaHCO}_{3}\right.$ 0,5 mol L-1) & $\begin{array}{r}0 \\
3 \\
13\end{array}$ & $\begin{array}{l}14 a \\
22 a \\
20 a\end{array}$ & $\begin{array}{l}14 \mathrm{~b} \\
23 \mathrm{a} \\
20 \mathrm{ab}\end{array}$ & $\begin{array}{l}15 a \\
24 a \\
21 a\end{array}$ & $\begin{array}{l}14 c \\
29 a \\
25 b\end{array}$ & $\begin{array}{l}14 b \\
30 a \\
26 a\end{array}$ \\
\hline $\mathrm{PO}_{\text {hid }}\left(\mathrm{NaOH} 0,1 \mathrm{~mol} \mathrm{~L}^{-1}\right)$ & $\begin{array}{r}0 \\
3 \\
13\end{array}$ & $\begin{array}{l}89 a \\
85 a \\
84 a\end{array}$ & $\begin{array}{l}93 a \\
84 a \\
84 a\end{array}$ & $\begin{array}{r}87 \mathrm{~b} \\
94 \mathrm{~b} \\
108 \mathrm{a}\end{array}$ & $\begin{array}{l}100 \mathrm{c} \\
117 \mathrm{~b} \\
124 \mathrm{a}\end{array}$ & $\begin{array}{l}104 a \\
117 a \\
126 a\end{array}$ \\
\hline POhid05 $(\mathrm{NaOH}$ 0,5 mol L-1) & $\begin{array}{r}0 \\
3 \\
13\end{array}$ & $\begin{array}{l}36 a \\
19 a \\
29 a\end{array}$ & $\begin{array}{l}49 a \\
26 a \\
37 a\end{array}$ & $\begin{array}{l}42 a \\
23 a \\
30 a\end{array}$ & $\begin{array}{l}35 a \\
22 b \\
27 a b\end{array}$ & $\begin{array}{l}25 a \\
26 a \\
23 a\end{array}$ \\
\hline
\end{tabular}


extratores atuam sobre as mesmas formas de $P$ inorgânico, como relatado por Tiessen et al. (1984), Cross \& Schlesinger (1995), Linquist et al. (1997), Friessen et al. (1997), Guo \& Y ost (1998).

Parte do $\mathrm{Pi}_{\text {hid }}$ foi dessorvida quando das extrações sucessivas com RTA, indicando que este métodoage dessorvendo formas de $\mathrm{P}$ obedecendo a sua energia de ligação, na ordem $\mathrm{Pi}_{\mathrm{RTA}}, \mathrm{Pi}_{\text {bic }}$ e Pi $\mathrm{Pi}_{\text {hid. }}$. Esses resultados concordam com os de Rheinheimer et al. (2000), que observaram que $20 \%$ do P dessorvido pelas extrações sucessivas com RTA foi proveniente da fração $P \mathrm{i}_{\text {bic }}$ e 79 \% das frações $P \mathrm{P}_{\text {hid }}$ e $\mathrm{Pi}$ hid05.

A fração $\mathrm{Pi}_{\mathrm{HCl}}$ foi pouco modificada pelas extrações, como ocorreu com os demais extratores (Quadros 1, 2 e 3). Esses resultados indicam que esta forma não compõea fração disponível nestetipo de sol o ou é inexpressiva como fonte de $\mathrm{P}$, mesmo em solo com disponi bilidade baixa, comojá foi observado por Gatiboni (2003).

Houve pequena depleção do $\mathrm{P}_{\text {res }}$ e aumento na fração $\mathrm{Pi}_{\text {hid05, }}$, mostrando que as extrações sucessivas com RTA provocaram a saída de $\mathrm{P}$ da fração mais recal citrante do solo e sua readsorção em formas de labilidade intermediária (Quadro 3).

As formas orgânicas de $P$ também foram modificadas pelas extrações sucessivas com RTA, havendo aumento nas frações $\mathrm{Po}_{\mathrm{bic}}$ e $\mathrm{Po}_{\mathrm{hid}}$ e pequena diminuiçãona fração $\mathrm{P}_{\text {hid05, }}$, principalmente nos solos com maior adição do fertilizante fosfatado (Quadro 3). Como o aumento nas frações mais lábeis de Po geralmente foi maior quea dessorção do $\mathrm{PO}_{\text {hid05, }}$, parte do $P$ o foi proveniente da fração $P_{\text {res }}$ que contém $P$ orgânico de alta recal citrância.

Pel os resultados mostrados, observa-se que as extrações sucessivas com RTA provocam modificações em todas formas de P do solo, dessorvendo as formas mais lábeis, mas promovendo reposição, mesmo que parcial, desses teores a partir de formas de menor labilidade, comotambém observado por Sharpley \& Smith (1985), Guo \& Yost (1998) e Gatiboni (2003) após o cultivo com plantas.

Para facilitar a comparação das modificações provocadas pel as extrações sucessivas, as formas de $P$ do sol o foram agrupadas em duas: o $\mathrm{P}$ geoquímico eoP biológico, como sugerido por Cross \& Schlesinger (1995). O P geoquímico é a soma das formas inorgânicas mais o P residual, enquanto o P biológico é a soma das frações orgânicas. O P geoquímico, o biológico e o somatório de todas as frações do fracionamento são apresentados no quadro 4 . Não houve diferenças $(P<0,05)$ do $P$ geoquímico com as extrações sucessivas, embora tenha sido observada

Quadro 4. Alterações dos teores de fósforo geoquímico, biológico e total do solo depois de extrações sucessivas com Mehlich-1, Mehlich-3 e resina trocadora de ânions

\begin{tabular}{|c|c|c|c|c|c|c|c|}
\hline \multirow{2}{*}{ F ração de $P$} & \multirow{2}{*}{$\begin{array}{c}\text { Extrator } \\
\text { prévio }\end{array}$} & \multirow{2}{*}{$\begin{array}{l}\text { Número } \\
\text { extração }\end{array}$} & \multicolumn{5}{|c|}{ Dose de $\mathrm{P}_{2} \mathrm{O}_{5}\left(\mathrm{~kg} \mathrm{ha}^{-1}\right)$} \\
\hline & & & $\mathbf{0}$ & 180 & 360 & 540 & 720 \\
\hline & & & & 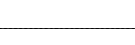 & $\mathrm{mg} \mathrm{kg}^{-1}$ & 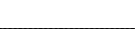 & 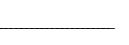 \\
\hline \multirow[t]{3}{*}{ P geoquímico } & Mehlich-1 & $\begin{array}{l}0^{(2)} \\
3 \\
13\end{array}$ & $\begin{array}{l}586 \mathrm{a}^{(1)} \\
572 \mathrm{aB} \\
511 \mathrm{aB}\end{array}$ & $\begin{array}{l}620 \mathrm{a} \\
598 \mathrm{aC} \\
574 \mathrm{aA}\end{array}$ & $\begin{array}{l}632 a \\
630 a B \\
603 a A\end{array}$ & $\begin{array}{l}700 a \\
674 \text { abA } \\
644 \text { bAB }\end{array}$ & $\begin{array}{l}737 a \\
680 a A \\
645 a A B\end{array}$ \\
\hline & Mehlich-3 & $\begin{array}{r}0 \\
3 \\
13\end{array}$ & $\begin{array}{l}586 \text { a } \\
645 \text { aA } \\
598 \text { aA }\end{array}$ & $\begin{array}{l}620 \text { a } \\
667 \text { aA } \\
615 \text { aA }\end{array}$ & $\begin{array}{l}632 a \\
648 a A \\
660 a A\end{array}$ & $\begin{array}{l}700 \mathrm{a} \\
713 \mathrm{aA} \\
673 \mathrm{aA}\end{array}$ & $\begin{array}{l}737 a \\
720 a A \\
665 a A\end{array}$ \\
\hline & RTA & $\begin{array}{r}0 \\
3 \\
13\end{array}$ & $\begin{array}{l}586 a \\
577 a \mathrm{a} \\
569 \mathrm{aB}\end{array}$ & $\begin{array}{l}620 \mathrm{a} \\
635 \mathrm{aB} \\
567 \mathrm{aA}\end{array}$ & $\begin{array}{l}632 a \\
616 a \mathrm{a} \\
585 \mathrm{aA}\end{array}$ & $\begin{array}{l}700 \mathrm{a} \\
654 \mathrm{abA} \\
602 \mathrm{bB}\end{array}$ & $\begin{array}{l}737 a \\
652 a A \\
597 a B\end{array}$ \\
\hline \multirow[t]{3}{*}{ P biológico } & Mehlich-1 & $\begin{array}{r}0 \\
3 \\
13\end{array}$ & $\begin{array}{l}139 a \\
114 a A \\
136 a A\end{array}$ & $\begin{array}{l}156 \mathrm{a} \\
173 \mathrm{aA} \\
172 \mathrm{aA}\end{array}$ & $\begin{array}{l}144 \mathrm{a} \\
169 \mathrm{aA} \\
147 \mathrm{aA}\end{array}$ & $\begin{array}{l}150 \mathrm{~b} \\
200 \mathrm{aA} \\
170 \mathrm{abA}\end{array}$ & $\begin{array}{l}143 \mathrm{a} \\
201 \mathrm{aA} \\
182 \mathrm{aA}\end{array}$ \\
\hline & Mehlich-3 & $\begin{array}{r}0 \\
3 \\
13\end{array}$ & $\begin{array}{l}139 \mathrm{a} \\
126 \mathrm{aA} \\
114 \mathrm{bB}\end{array}$ & $\begin{array}{l}156 \mathrm{a} \\
147 \mathrm{abB} \\
126 \mathrm{bC}\end{array}$ & $\begin{array}{l}144 \mathrm{a} \\
127 \mathrm{aB} \\
118 \mathrm{aB}\end{array}$ & $\begin{array}{l}150 \mathrm{a} \\
130 \mathrm{bC} \\
133 \mathrm{bB}\end{array}$ & $\begin{array}{l}143 \mathrm{a} \\
118 \mathrm{aC} \\
131 \mathrm{aB}\end{array}$ \\
\hline & RTA & $\begin{array}{r}0 \\
3 \\
13\end{array}$ & $\begin{array}{l}139 a \\
126 a A \\
133 a A B\end{array}$ & $\begin{array}{l}156 \mathrm{a} \\
133 \mathrm{bC} \\
140 \mathrm{bB}\end{array}$ & $\begin{array}{l}144 \mathrm{a} \\
141 \mathrm{aB} \\
158 \mathrm{aA}\end{array}$ & $\begin{array}{l}150 \mathrm{~b} \\
168 \mathrm{aB} \\
176 \mathrm{aA}\end{array}$ & $\begin{array}{l}143 \mathrm{a} \\
174 \mathrm{aB} \\
175 \mathrm{aA}\end{array}$ \\
\hline \multirow[t]{3}{*}{ Somatório total } & Mehlich-1 & $\begin{array}{r}0 \\
3 \\
13\end{array}$ & $\begin{array}{l}725 a \\
686 \text { aB } \\
646 a A\end{array}$ & $\begin{array}{l}776 \mathrm{a} \\
771 \mathrm{aB} \\
746 \mathrm{bA}\end{array}$ & $\begin{array}{l}776 \text { a } \\
799 \text { aA } \\
750 \text { aA }\end{array}$ & $\begin{array}{l}850 \mathrm{ab} \\
874 \mathrm{aA} \\
814 \mathrm{bA}\end{array}$ & $\begin{array}{l}880 a \\
882 a A \\
826 a A\end{array}$ \\
\hline & Mehlich-3 & $\begin{array}{r}0 \\
3 \\
13\end{array}$ & $\begin{array}{l}725 \mathrm{a} \\
771 \mathrm{aA} \\
711 \mathrm{aA}\end{array}$ & $\begin{array}{l}776 \mathrm{a} \\
814 \mathrm{aA} \\
741 \mathrm{aA}\end{array}$ & $\begin{array}{l}776 \text { a } \\
801 \text { aA } \\
778 \text { aA }\end{array}$ & $\begin{array}{l}850 a \\
843 a b A \\
806 b A\end{array}$ & $\begin{array}{l}880 a \\
838 \text { aA } \\
796 a A B\end{array}$ \\
\hline & RTA & $\begin{array}{r}0 \\
3 \\
13\end{array}$ & $\begin{array}{l}725 a \\
702 \text { aB } \\
702 \text { aA }\end{array}$ & $\begin{array}{l}776 \mathrm{a} \\
767 \mathrm{aB} \\
708 \mathrm{aA}\end{array}$ & $\begin{array}{l}776 a \\
757 \text { aB } \\
743 a A\end{array}$ & $\begin{array}{l}850 \text { a } \\
822 \text { aA } \\
778 \text { aA }\end{array}$ & $\begin{array}{l}880 \mathrm{a} \\
826 \mathrm{aA} \\
771 \mathrm{aB}\end{array}$ \\
\hline
\end{tabular}

(1) Médias seguidas da mesma letra, minúsculas, entre as extrações em cada método, e maiúsculas, entre os métodos em cada extração, não diferem entre si pel o teste DMS $(P<0,05) .{ }^{(2)}$ Solo original sem extrações sucessivas previamente ao fracionamento. 
diminuição nos teores após as extrações. Para o P biológico também houve pouca diferenciação estatística entre os teores antes e depois das extrações sucessivas. Houve diminuição média de 9,2, 3,4 e $10,4 \%$ do $P$ geoquími co com as extrações sucessivas com Mehlich-1, Mehlich-3 e RTA, respectivamente. Por outro lado, o P biológico aumentou, em média, 10,2 e 7,0 \%, com as extrações por Mehlich-1 e RTA, e diminuiu $14,8 \%$ com o uso de Mehlich-3. Isso indica que, enquanto os extratores Mehlich-1 eRTA agem exclusivamente sobre as formas inorgânicas de $P$, o Mehlich-3, em razão de sua composição, exerce ação conjugada, dessorvendo $\mathrm{P}$ de formas inorgânicas e orgânicas.

O somatório de todas as formas de $\mathrm{P}$ do fracionamento de Hedley (Quadro 4) mostra que o mesmo foi sensível às extrações sucessivas, havendo diminuição média de 5,6, 4,2 e 7,2 \% no teor de P após a utilização de Mehlich-1, Mehlich-3 e RTA, respectivamente. No entanto, pelo fato de o teor total de $\mathrm{P}$ ser muito superior ao extraído pelos métodos, não houve, para a maioria dos casos, significância estatística da depleção observada.

\section{CONCLUSÕES}

1. Os métodos Mehlich-1 e resina trocadora de ânions extraíram $\mathrm{P}$ de formas inorgânicas, enquanto o método Mehlich-3 teve ação conjugada, dessorvendo $\mathrm{P}$ tanto de formas inorgânicas como de orgânicas.

2. A ordem de dessorção de $P$ dos extratores Mehlich-1, Mehlich-3 eRTA foi similar, obedecendo sua labilidade no solo, seguindo a seqüência das formas mais lábeis para as de menor labilidade.

\section{LITE RATURA CITADA}

ADEPETU, J.A. \& COREY, R.B. Organic phosphorus as a predictor of plant-available phosphorus in soils of southern Nigeria. Soil Sci., 122:159-164, 1976.

BARROW, N.J . Reactions with variable-charge soils. Dordrecht, Martinus Nijhoff., 1987. 191p.

BAYER, C. Dinâmica da matéria orgânica em sistemas de manejo de solos. Porto Alegre, Universidade Federal do Rio Grande do Sul, 1996. 241p. (Tese de Doutorado)

BROOKES, P.C. \& POWLSON, D.S. Preventing phosphorus losses during perchloric acid digestion of sodium bicarbonate soil extracts. J. Sci. Food Agric., 32:671-674, 1981.

CAJ USTE, L.J . \& KUSSOW, W.R. Use and limitations of the North Carolina method to predict available phosphorus in some Oxisols. Trop. Agric., 51:246-252, 1974.
CAMPELLO, M.R.; NOVAIS, R.F.; FERNANDEZ, I.E.R.; FONTES, M.P.F. \& BARROS, N.F. Avaliação da reversibilidade de fósforo não-lábil para lábil em solos com diferentes características. R. Bras. Ci. Solo, 18:157-165, 1994.

CONDRON, L.M.; GOH, K.M. \& NEWMAN, R.H. Nature and distribution of soil phosphorus as revealed by a sequential extraction method followed by 31P nuclear magnetic resonance analysis. J. Soil Sci., 36:199-207, 1985.

CROSS, A.F. \& SCHLESINGER, W.H. A literature review and evaluation of the Hedley fractionation: Applications to the biogeochemical cycle of soil phosphorus in natural ecosystems. Geoderma, 64:197-214, 1995.

DALAL, R.C. Soil organic phosphorus. Adv. Agron., 29:83-117, 1977.

DICK, W.A. \& TABATABAI, M.A. Determination of orthophosphate in aqueous solutions containing labile organic and inorganic phosphorus compounds. J . Environ. Qual., 6:82-85, 1977.

FIXEN, P.E. \& GROVE, J.H. Testing soils for phosphorus. In: WESTERMAN, R.L., ed. Soil testing and plant analysis, 3.ed. Madison., Soil Science Society of America, 1990. p.141180.

FRIESEN, D.K.; RAO, I.M.; THOMAS, R.J .; OBERSON, A. \& SANZ, J .I. Phosphorus acquisition and cycling in crop and pasture systems in low fertility tropical soils. Plant Soil, 196:289-294, 1997.

GATIBONI, L.C. Disponibilidade de formas de fósforo do solo às plantas. Santa Maria, Universidade Federal de Santa Maria, 2003. 231p. (Tese de Doutorado)

GATIBONI, L.C.; KAMINSKI, J .; RHEINHEIMER, D.S. \& SAGGIN, A. Quantificação do fósforo disponível por extrações sucessivas com diferentes extratores em Latossolo Vermelho distroférrico. R. Bras. Ci. Solo, 26:1023-1029, 2002.

GUO, F. \& YOST, R.S. Partitioning soil phosphorus into three discrete pools of differing availability. Soil Sci., 163:822833, 1998.

HEDLEY, M.J .; STEWART, J .W.B. \& CHAUHAN, B.S. Changes in inorganic and organic soil phosphorus fractions induced by cultivation practices and by laboratory incubations. Soil Sci. Soc. Am. J ., 46:970-976, 1982.

LINQUIST, B.A.; SINGLETON, P.W. \& CASSMAN, K.G. Inorganic and organic phosphorus dynamics during a buildup decline of avaliable phosphorus in a Ultisol. Soil Sci., 162:254-264, 1997.

MAROKO, J.B.; BURESH, R.J . \& SMITHSON, P.C. Soil phosphorus fractions in unfertilized fallow-maize systems on twotropical soils. Soil Sci. Soc. Am. J ., 63:320-326, 1999.

MCKEAN, S.J . \& WARREN, G.P. Determination of phosphate desorption characteristics in soils using successive resin extractions. Comm. Soil Sci. Plant Anal., 27:2397-2417, 1996. 
MEHLICH, A. Determination of $\mathrm{P}, \mathrm{Ca}, \mathrm{Mg}, \mathrm{K}, \mathrm{Na}$ and $\mathrm{NH}_{4}$ by North Carolina Soil Testing Laboratoris. Raleigh, University of North Carolina, 1953. não publicado.

MEHLICH, A. Mehlich-3 soil test extractant: A modification of Mehlich-2 extractant. Comm. Soil Sci. Plant Anal., 15:14091416, 1984.

MURPHY, J \& \& RILEY, J.P. A modified single solution method for the determination of phosphate in natural waters. Anal. Chim. Acta, 27:31-36, 1962.

NOVAIS, R.F. \& SMYTH, T.J . Fósforo em solo e planta em condições tropicais. Viçosa, Universidade F ederal deViçosa, 1999. 399p.

PARFITT, R.L. Anion adsorption by soils and soil materials. Adv. Agron., 30:01-46, 1978.

RHEINHEIMER, D.S.; ANGHINONI, I. \& KAMINSKI, J . Depleção do fósforo inorgânico de diferentes frações provocadas pela extração sucessiva com resina em diferentes solos e manejos. R. Bras. Ci. Solo, 24:345-354, 2000.

SILVA, F.C. \& RAIJ , B. van. Disponibilidade de fósforo em solos avaliada por diferentes extratores. Pesq. Agropec. Bras., 34:267-288, 1999.
SHARPLEY, A.N. \& SMITH, S.J . Fractionation of inorganic and organic phosphorus in virgin and cultivated soils. Soil Sci. Soc. Am. J ., 49:127-130, 1985.

SHARPLEY, A.N.; TIESSEN, H. \& COLE, C.V. Soil phosphorus forms extracted by soil tests as a function of pedogenesis. Soil Sci. Soc. Am. J ., 51:362-365, 1987.

STRECK, E.V.; KÄMPF, N.; DALMOLIN, R.S.D.; KLAMT, E. NASCIMENTO, P.C. \& SCHNEIDER, P. Solos do Rio Grande do Sul. Porto Alegre, Universidade Federal do Rio Grande do Sul, 2002. 107p.

TATE, K.R. \& NEWMAN, R.K. Phosphorus fractions of a climosequence of soils in New Zeland tussock gassland.. Soil Biol. Biochem., 14:191-196, 1982.

TEDESCO, M.J .; GIANELLO, C.; BISSANI, C.A.; BOHNEN, H. \& VOLKWEISS, S.J. Análise de solo, plantas e outros materiais. Porto Alegre, Universidade Federal do Rio Grande do Sul, 1995. 174p.

TIESSEN, H.; STEWART, J .W.B. \& COLE, C.V. Pathways of phosphorus transformations in soils of differing pedogenesis. Soil Sci. Soc. Am. J ., 48:853-858, 1984.

UNITED STATES ENVIRONMENTAL PROTECTION AGENCY - USEPA. Methods of chemical analysis for water and wastes. Cincinnati: USEPA, 1971. 312p. 
Luciano Colpo Gatiboni et al.

R. Bras. Ci. Solo, 29:363-371, 2005 